\title{
Non-Specific Therapy.
}

\section{By Dr. J. Stephenson.}

I $\mathrm{N}$ inoculation against typhoid fever, dead typhoid bacilli are injected subcutaneously or into a muscle. Inoculation against plague consists in the injection of an emulsion of dead plague bacilli artificially grown in broth. These are prophylactic measures for the protection of persons who are likely to be exposed to infection. Hydrophobia vaccine, used in order to prevent the development of the disease in persons who have been bitten by a rabid animal, is prepared according to a definite system from the spinal cords of rabbits inoculated with the disease. Antidiphtheritic serum, used in the treatment of patients actually suffering from diphtheria, is the blood-serum of a horse which has had diphtheria toxin (the broth in which diphtheria bacilli have been grown, and from which the bacilli have been filtered off) repeatedly injected into it.

In using vaccines the object is to stimulate the individual to produce protective substances in his own body; in using sera, the protective substances, elaborated by some other animal, are themselves supplied to the patient. But in all the above instances, and in numerous other similar modes of treatment,whether the treatment takes place before infection (i.e. is prophylactic), or is carried out during the incubation period, or during the actual disease; whether bacilli are used, or a serum free from bacilli but containing an antitoxin,--the implication is that certain substances are protective against one disease, certain other substances against another; in other words, the treatment is specific. The idea of specificity may go even further, as where a patient is treated by means of the particular strain of microorganism, or the particular mixture of them, that he himself harbours (the use of autogenous vaccines in aşthma, acne, boils, etc.). As Sir Almroth Wright has recently written :-

"That immunisation is always strictly specific counts as an article of faith; and it passes as axiomatic that microbic infections can be warded off only by working with homologous vaccines, and that we must in every case, before employing a vaccine therapeutically, make sure that the patient is harbouring the corresponding microbes."

To attempt treatment on a non-specific basis would seem therefore at first glance to be a step backward, and investigation of such a subject illogical, if nothing worse. But while reason, working on the accepted lines, was all against the idea, facts have cropped up repeatedly which seem to give a value to non-specific treatment. At first these were ignored; but a time has come when this method of disposing of them is no longer possible. Thus, to quote Wright again :-

"I confess to having shared the conviction that immunisation is always strictly specific. Twenty years ago, when it was alleged, before the Indian Plague Commission, that anti-plague inoculation had cured eczema, gonorrhoea, and other miscellaneous infections, I thought the matter undeserving of examination. I took the same view when it was reported in connection with anti-typhoid inoculation that it rendered the patients much less susceptible to malaria. Again, some years ago, when applying No. 2744 , VOL. IO9] pneumococcus inoculations as a preventive against pneumonia in the Transvaal mines, I nourished exactly the same prejudices. But here the statistical results which were obtained in the Premier Mine demonstrated that the pneumococcus inoculations had, in addition to bringing down the mortality from pneumonia by 85 per cent., reduced also the mortality from "other diseases" by 50 per cent. From that on we had to take up into our categories the fact that inoculation produces in addition to "direct" also " collateral" immunisation. This once recognised, presumptive evidence of collateral immunisation began gradually to filter into our minds. ... From such cases hints are conveyed to us that there may exist a useful sphere of application for collateral immunisation ;... we should discard the confident dogmatic belief that immunisation should be strictly specific, and that we should in every case of failure endeavour to make our immunisation more and more strictly specific. We should instead proceed upon the principle that the best vaccine to employ will always be the vaccine which gives on trial the best immunising response against the microbe we propose to combat."

The present position of non-specific therapy is explained in a recently published volume by Dr. Petersen of Chicago, ${ }^{1}$ from which the above quotation from Wright is taken. When we come to inquire into the rationale of the procedure, we find that a theoretical basis to account for the results has been lacking,-the treatment has been empirical. With Petersen, we may perhaps put the matter broadly thus: the reaction of the body is fundamentally the same in all cases of injury ; there is an effort to dilute the noxious agent (increased flow of lymph), to remove it (phagocytosis by leucocytes), to neutralise it (manufacture of antibodies); these failing, to wall it in. We may, in trying to influence this process, adopt one of two avenues of approach ; we may proceed against the cause of the inflammatory reaction by fostering the production of an antibacterial agent or an antitoxin; treatment on these lines must necessarily be specific, must be directed against the particular microorganism-or toxin. Or we may endeavour to alter the inflammatory reaction of the body itself,--to stimulate the potential forces, latent or held in abeyance until the non-specific stimulation brings them into activity. This is somewhat vague; Wright has, in a recent lecture (see the Lancet, May 6), described one way, at least, in which the activation works. He has discovered that "the intravenous injection of a vaccine is immediately followed by the appearance of bactericidal substances in the blood, which are not specific but can act upon various types of organisms. . The late result of an inoculation with, say, a typhoid vaccine, is the production of antibodies which are specific for the typhoid bacillus, but the immediate result of such an inoculation is the appearance of nonspecific antibodies. Inasmuch as leucocytes possess the power of inhibiting the growth of organisms on culture media, it seems likely that these non-specific substances exist ready-formed in the leucocytes which yield them in response to the immediate demand."

1 Protein Therapy and Nonspecific Resistance. By Dr. Williatn F Petersen. Pp. xvili $+3 I_{4}$. (New York: The Macmillan Company; London: Macmillan and Co., Ltd., I922.) 2 Is. net. 
Already a large number of substances have been used as non-specific agents ; in many cases, of course, these remedies were employed long before any explanation of their action had been formulated on the above lines. Of the long list of agents given by Petersen only a few can be mentioned.

First comes counter-irritation by means of thermocautery, seton, blisters, etc. Each of these procedures has for object the production of a focus of inflammatory exudation, suppuration, or necrosis ; the absorption of the pathological exudates must lead to a tissue stimulation similar to that which follows more modern non-specific injections. Our non-specific therapy is thus but part and parcel of this older practice of counterirritation.

Normal animal sera have been used,-horse, beef, goat, sheep, chicken, and other sera; these were first injected subcutaneously, and in more recent years into a vein; as much as 250 c.c. of beef serum have been given in anthrax without injury. Antibacterial sera and antitoxins have been widely used,-diphtheria and tetanus antitoxin, antistreptococcic, antipneumococcic, antidysenteric serum, etc.;-as remedies, that is, not in the homologous diseases, but in other morbid conditions, e.g. diphtheria antitoxin against streptococcus infection, tuberculosis, lupus, etc. The numerous vaccines, prepared in the first place as specific agents,-typhoid, dysentery, streptococcal, pneumococcal, influenza vaccines, - have also been used with a non-specific object.

Various native proteins have been given,--solution of egg albumen and serum albumen injected subcutaneously, milk by intramuscular injection, casein, gelatin ; of protein split products, proteoses (albumoses) prepared from different proteins sometimes give a very. prompt and satisfactory reaction. The enzyme treatment of cancer, exploited some years ago, consisted in the subcutaneous injection of a trypsin solution; a general reaction,-chill, sweating, and rise of temperature,-followed the injection, and the patient would have several days of comparative comfort.

Colloidal metals constitute another group of remedies; these are active catalytic agents, and it is supposed that they act therapeutically in virtue of this property as inorganic ferments; they whip up the organism, which responds, if response is possible, by producing more leucocytes. A number of colloidal metals have been prepared for therapeutic employment; the colloidal silver preparations have been in use longest, but arsenic, zinc, gold, manganese, iron, mercury, and other metals, as well as sulphur and iodine, have been employed with varying success in septic conditions, endocarditis, rheumatism, trench fever, etc.

The use of light, Röntgen rays, and radium must also be mentioned. These agents first stimulate tissue cells, and later, with prolonged exposure, cause their death. In both cases substances enter the blood stream which produce a general reaction; this may be mild, or accompanied by severe fever. After moderate reactions of this type, if the patient is in good condition and able to respond, improvement of appetite, nutrition, and general well-being may set in, just as after other non-specific agents. Here, then, we have at least a partial explanation of the effect of heliotherapy in tuberculosis, as used, for example, at Leysin in Switzerland, of which the public has heard much in the last few months.

- About half of Dr. Petersen's book is occupied with an account of the methods used and the results obtained in the numerous diseases for which non-specific therapy has been tried. The last chapter, on indications and contra-indications, gives much useful advice. We are reminded that the method can only be applied intelligently if we recognise that by it all the forces of cellular and humoral resistance are for a short period of time keyed to the highest pitch; stimulation of this kind is useless when the cells of the body are profoundly fatigued, and hence injections must be given early in the course of the disease. But "the non-specific method of treatment should under no circumstances be considered as a rival or a substitute for the proven specific measures that we have at our command. That a non-specific factor is at times and possibly often associated with the specific reaction may be true, the more reason that both should be studied and both utilised in their proper time and place."

The bibliography runs to no less than fifty pages, and must, one would think, be complete up to date. The book as a whole forms an interesting, convenient, and comprehensive account of a recent development of medical thought and practice.

\section{The Solvay Institute of Chemistry.}

$\mathrm{T}^{\mathrm{H}}$ IE first meeting of the "Institut International de Chimie Solvay" was held in Brussels on April. 20-27, under the presidency of Sir William Pope. It will be remembered that before the war M. Ernest Solvay set aside a capital sum to be expended in the course of thirty years by the International Institute of Physics, and that meetings under the auspices of this Institute have been held in Brussels both before and since the war. More recently M. Solvay has set aside a further capital sum of one million francs, also to be expended in thirty years, for the promotion of the science of chemistry.

The meetings of the Institute are attended by delegates from different countries, the number being limited to about thirty, so that the discussions may be as free and as informal as possible. The recent meeting was devoted to the consideration of a number of those questions which affect the foundations of modern chemistry, and its programme included the presentation of papers on isotopes, by Soddy, by Aston, and by Perrin and Urbain ; on X-ray analysis and molecular structure, by W. H. Bragg; on the electronic theory of valency, by Mauguin ; on optical activity, by Pope and by Lowry; and on chemical mobility, by Job.

In connection with the papers on isotopes, considerable discussion was aroused as to the possibility of two dissimilar arrangements of planetary electrons around the same type of nucleus. The possibility of such an isomerism in the external domain of the atom was conceded, although at present only as a hypothesis;

NO. 2744 , VOL. IOg] 